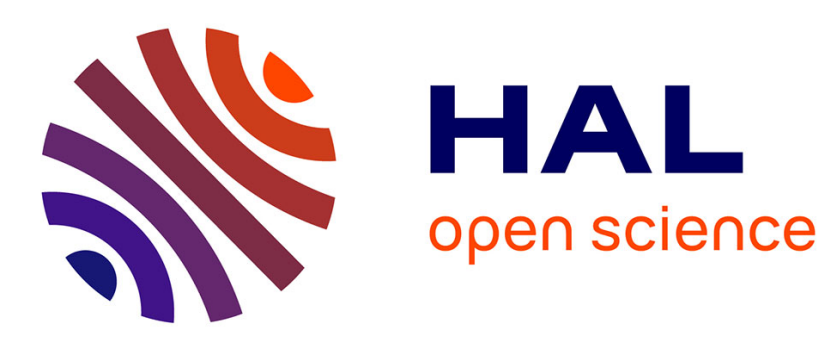

\title{
Les techniques vinicoles grecques, des vendanges aux Anthestéries: nouvelles perspectives \\ Thibaut Boulay
}

\section{To cite this version:}

Thibaut Boulay. Les techniques vinicoles grecques, des vendanges aux Anthestéries: nouvelles perspectives. Dialogues d'histoire ancienne, 2012, Supplément 7, pp.95-115. 10.3406/dha.2012.3531 . hal-01263943

\section{HAL Id: hal-01263943 \\ https://hal.science/hal-01263943}

Submitted on 28 Jan 2016

HAL is a multi-disciplinary open access archive for the deposit and dissemination of scientific research documents, whether they are published or not. The documents may come from teaching and research institutions in France or abroad, or from public or private research centers.
L'archive ouverte pluridisciplinaire HAL, est destinée au dépôt et à la diffusion de documents scientifiques de niveau recherche, publiés ou non, émanant des établissements d'enseignement et de recherche français ou étrangers, des laboratoires publics ou privés. 


\section{Les techniques vinicoles grecques, des vendanges aux Anthestéries : nouvelles perspectives}

Thibaut Boulay

\section{Résumé}

Le présent travail se propose d'examiner les techniques vinicoles développées par les praticiens grecs visant à maîtriser le processus fermentaire, depuis le foulage des raisins jusqu'à l'entonnage des pithoi, ainsi que les modalités d'un élevage des vins sous atmosphère oxydative. La grande fête dionysiaque des Anthestéries pourrait être mise en rapport avec la formation, à la fin de l'hiver, d'un voile de levures à la surface du vin, que les Grecs surnommaient la 'fleur' du vin. Cette approche technique permet enfin de reprendre sur de nouveaux frais le commentaire du célèbre décor du stamnos de Nocera.

\section{Abstract}

Greek wine making techniques, from harvest time to the Anthesteria: new perspectives.

The present paper examines the winemaking techniques developed by Greek practitioners to control the fermentation process, from crushing the grapes to the casking of pithoi, and the methods of wine maturing under oxidizing atmosphere. The three-day-long Dionysian festival of the Anthesteria could be related with the formation, at the end of the winter, of a voile or film of yeast on the surface of the wine, which the Greeks called the "flower" of the wine. Finally, this technical approach enables us to suggest a new commentary of the famous scene of the so-called stamnos of Nocera.

\section{Citer ce document / Cite this document :}

Boulay Thibaut. Les techniques vinicoles grecques, des vendanges aux Anthestéries : nouvelles perspectives. In: Dialogues d'histoire ancienne. Supplément $n^{\circ} 7,2012$. L'histoire de l'alimentation dans l'Antiquité. Bilan historiographique. Journée de printemps de la SOPHAU - 21 mai 2011. pp. 95-115.

doi : 10.3406/dha.2012.3531

http://www.persee.fr/doc/dha_2108-1433_2012_sup_7_1_3531

Document généré le 05/01/2016 


\title{
Les techniques vinicoles grecques, des vendanges aux Anthestéries : nouvelles perspectives
}

Thibaut Boulay

Université François Rabelais (Tours)

L'Équipe Alimentation (LÉA, Tours)

\begin{abstract}
«Selon moi, c'est l'oxygène qui fait le vin ; c'est par son influence que le vin vieillit ; c'est lui qui modifie les principes acerbes du vin nouveau et en fait disparaître le mauvais goût ; c'est encore lui qui provoque les dépôts de bonne nature dans les tonneaux et dans les bouteilles, et loin, par exemple, qu'une absorption de quelques centimètres cubes de gaz oxygène par litre de vin use ce vin, lui enlève son bouquet et l'affaiblisse, je crois que le vin n'est pas arrivé à sa qualité et ne doit pas être mis en bouteille tant qu'il n'a pas absorbé une quantité d'oxygène bien supérieure à celui-ci. »

Louis Pasteur. Études sur le vin, ses maladies, causes qui les provoquent, procédés nouveaux pour le conserver et pour le vieillir, Paris, 1866, p. 90
\end{abstract}

Les Grecs, comme tous les vinificateurs jusqu'à Pasteur, ignoraient tout de la fermentation alcoolique et des nombreuses réactions biochimiques, catalysées par les enzymes de micro-organismes, qui déterminent les arômes et le goût du vin ${ }^{1}$. Mais si la vinification est le résultat de l'évolution spontanée d'un système microbien complexe dans le jus de raisin, transformation qui revêtait un caractère sacré, les Grecs n'adoptaient pas, face à ce phénomène,

* J'adresse mes vifs remerciements à Aline Lonvaud, Professeur d'OEnologie à l'université Victor Segalen-Bordeaux II, qui m’a généreusement prodigué ses précieuses remarques et observations. Les hypothèses ici présentées n'engagent naturellement que ma seule responsabilité.

1 Voir Dubourdieu et al. 2004 et Lonvaud-Funel et al. 2010. 
une attitude passive. Pline admettait même que les Grecs « avaient édicté des règles spéciales pour apprêter le vin et en ont fait un art », citant les noms de quatre célèbres détenteurs d'un savoir œnologique, Euphronios, Aristomachos, Kommiadès et Hikésios ${ }^{2}$. Ces règles, améliorant la qualité de la transformation du moût de raisin en vin, témoignent d'une volonté manifeste de maîtriser le processus fermentaire. Dans cette contribution, je souhaiterais prolonger les observations pionnières de Marie-Claire Amouretti sur l'originalité technique du vin grec $^{3}$, et aborder en particulier la question des vinifications, depuis les vendanges jusqu'à la fête des Anthestéries, où l'on ouvrait traditionnellement les jarres de vin nouveau, et reprendre sur de nouveaux frais la question de la nature des vins produits ${ }^{4}$. Il sera ainsi nécessaire d'appréhender certaines techniques bien connues, comme le plâtrage et l'addition d'eau de mer, mais aussi celles, plus énigmatiques, qu'éclairent désormais les récentes recherches en microbiologie. Pour cela, je privilégierai l'étude des auteurs grecs, de Théophraste aux Géoponiques, aux agronomes latins, trop souvent sollicités, et parfois à contresens ${ }^{5}$. On ne peut plus étudier

2 Pl., $H N, 14.120$. Cet art est très lié aux pratiques médicales, puisque le vin était fréquemment utilisé comme remède ( $\phi \dot{\alpha} \rho \mu \alpha \kappa o v)$ ), cf. notamment Jouanna 1996. Qualités intrinsèques et vertus médicales apparaissent indissociables, comme le montre la genèse de certains grands crus grecs : Pline indique ainsi que le médecin Érasistrate a, au III ${ }^{\mathrm{e}}$ s. av. J.-C., grandement contribué à la réputation des vins de Thasos (Pl., $H N, 14.73)$ tandis que son frère Kléophante s'illustra par la théorie de l'emploi du vin, ses usages diététiques ou ses propriétés thérapeutiques (Pl., HN, 26.14). Columelle (Col., Rust., 1.1) et Varron (Varr., R., 1.1) ont utilisé les travaux de l'agronome athénien Euphronios. On ne sait rien de Kommiadès ou d'Andromachos, dont les traités sur la préparation du vin ne sont cités que par Pline (Pl., $H N$, 14.120 et 15 , index). Sur le médecin Hikésios, auteur d'un ouvrage de diététique, $\pi \varepsilon p i$ ü $\lambda \eta \varsigma$, connu presque uniquement par les citations d'Athénée, cf. Gourevitch 2000. Sur l'adoption des usages médicaux du vin en Italie à la fin de la République, voir également Tchernia, 1997.

3 Amouretti 1996.

4 Voir le chapitre consacré aux vinifications dans le monde romain par A. Tchernia dans Tchernia \& Brun 1999, 110-147, avec une dimension expérimentale.

5 Voir notamment les remarques de Boissinot 2003, p. 38. Notons cependant que Palladius, auteur convaincu des bienfaits de l'autarcie et défenseur d'une production agricole diversifiée, se rapproche davantage des usages grecs. 
les vins grecs à partir de recettes de contrefaçon ou de falsification de Caton, de Pline ou de Columelle.

Il faut d'emblée différencier les grands vins, qui sont des vins moelleux obtenus par concentration naturelle, comme le Thasos, le Chios, le Tmolos ou le Maronée ${ }^{6}$ des vins ordinaires, de consommation courante ${ }^{7}$. Les vins de la première catégorie étaient issus de raisins secs, séchés sur la souche ou sur des claies tandis que les vins de le seconde catégorie étaient issus de raisins également vendangés à maturité, mais qui n'avaient pas subi de traitement particulier. Le passerillage sur souche ou hors souche présente l'avantage d'enrichir le raisin en sucre, par concentration. Produisant une quantité bien inférieure de moût, 25 litres en moyenne pour $100 \mathrm{~kg}$ de raisin contre 65 à 75 litres en conditions normales, cette seconde technique s'inscrit dans une

6 Il faut renoncer à les qualifier de vins de liqueur, dont la fermentation est stoppée en cours de processus par l'adjonction d'alcool, pour éviter toute confusion. La recette donnée par Florentinus (Geop. 8.23) pour fabriquer du «vin de Thasos », qui donne à penser que l'on ajoutait du moût réduit et de l'eau de mer bouillie au moût, ne décrit pas la méthode traditionnelle comme semblent le croire Salviat 1986, 174-175 et Amouretti 1996, 56-57. Il s'agit d'une recette pour imiter le vin de Thasos, comme l'a bien vu Dalby 2010, p. 178, n. 4, dans son commentaire du passage : «this is a recipe for imitating the flavour ». Les expérimentations récentes montrent que ces recettes ne permettent pas d'imiter les vins à concentration naturelle de sucres, cf. les observations de Bouvier 2007, p. 16 (qui pourtant, p. 47, croit lui aussi que le Thasos est un vin liquoreux dont les Géoponiques nous donneraient le secret). D'ailleurs, les vins qui n'étaient pas apprêtés avaient la préférence des amateurs, cf. Col., Rust., 12.19.2 ou Pl., HN, 23.45. Le vin doux (glukismos) des collations offertes par les grands bienfaiteurs et évoquées dans les décrets honorifiques, comme les décrets de Priène pour Moschion (I. Priene, 108, 1. 257-259 et 272-274) ou pour Hérodès (I. Priene, 109, 1. 193-194), le décret de Colophon pour Polémaios (Robert \& Robert 1989, p. 15, col. IV, 1. 24-26) ou le décret de Kymè pour Kléanax, a souvent été interprété comme un vin muté par adjonction de moût réduit, peu alcoolisé (ainsi Amouretti, 1996, p. 57). Cette interprétation pose problème : dans ces conditions, il n'est guère possible d'inhiber les levures et donc de stopper la fermentation alcoolique, tout en conservant des sucres résiduels, puisque les Grecs ne connaissaient ni l'alcool, ni le dioxyde de soufre. Je reviendrai ailleurs sur cette question.

7 Voir les remarques générales de Bresson 2007, 128-134, qui distingue vins ordinaires et vins de qualité. 
démarche de recherche qualitative et de valorisation du produit ${ }^{8}$. Cette distinction faite, suivons à présent les techniques de vinification, depuis les suites des vendanges jusqu'à l'ouverture des jarres et la mise en amphores.

\section{Le foulage et pressurage}

Les vendanges ne commençaient que lorsque les grappes de raisin étaient parvenues à maturité 9 , de préférence en lune décroissante ${ }^{10}$. Hésiode recommandait déjà une récolte tardive suivie d'un passerillage de dix jours au soleil, puis de cinq jours à l'abri et à l'ombre ${ }^{11}$. Les Grecs ne recherchaient en aucune manière à récolter des raisins botrytisés ${ }^{12}$. Les conditions climatiques du bassin égéen interdisent en effet le développement d'une «pourriture noble ». En revanche, les vents chauds du début de l'automne favorisaient le dessèchement des raisins sur souche, dont on pouvait pincer les pédoncules ou tordre les sarments pour interrompre la circulation de la sève ${ }^{13}$.

Lorsqu'un passerillage sur claies ou sur tuiles, au soleil direct, était pratiqué, cinq à dix jours suffisaient. Les producteurs devaient seulement retourner les grappes ${ }^{14}$ et assurer un tri journalier pour éliminer les grappes ou les grains attaqués par les insectes, les moisissures et les pourrissements ${ }^{15}$, ce qui garantissait la présence minimale de germes d'altération potentielle. Dans

8 Notre méconnaissance des caractères propres aux cépages grecs, le pramnien de Smyrne, de Lesbos ou d'Ikaros ou bien le phanaios de Chios ou la mersitis d'Héraclée du Pont, ne nous permet guère de préciser les attentions particulières dont ils étaient l'objet. Salviat 1986, p. 186, veut voir, après d'autres, le muscat dans le cépage pramnien, sans argument décisif. Sur les cépages grecs, voir Salviat 1989.

9 Geop. 3.13.

10 Geop. 5.46.

11 Hes., Op., 609-614.

12 Je me démarque sur ce point de l'interprétation de Amouretti 1996, p. 56.

13 Geop. 5.52. Cf. Pl., $H N, 14.83-84$ et Pall. 11.22 (passerillage destiné à produire des raisins secs dont la finalité n'est pas la production de vin).

14 Pl., $H N, 14.77$, parle de trois fois par jour.

15 X., Oec., 19.8.

DHA supplément 7 
les régions les plus chaudes, un passerillage direct au soleil pouvait entraîner un phénomène préjudiciable de caramélisation des sucres ${ }^{16}$. À l'ombre, le passerillage devait durer deux à trois semaines. Il fallait également se garder d'un passerillage trop long: les moûts trop concentrés en sucres connaissent des fermentations laborieuses, avec un démarrage difficile et des arrêts. Dans ce cas cependant, il était alors possible d'accélérer le processus en ayant recours à des raisins frais.

Selon les cas, les grappes étaient foulées dans les vignes mêmes, comme cela semble avoir été le cas à Thasos, ou dans les chais ${ }^{17}$. Cette opération pouvait intervenir après une à trois journées d'attente du raisin dans l'aire de foulage ${ }^{18}$. Sous le poids de la vendange débutait alors une macération pelliculaire ou pré-fermentaire. Cette opération favorisait l'extraction des arômes et précurseurs d'arôme du raisin, à la condition que la vendange soit propre. L'éraflage était inutile puisque la maturation du raisin constituait le premier critère pour démarrer la vendange. Diophanès précise ainsi que le raisin est mûr si, en écrasant le grain, la pulpe n'adhère plus au pépin ${ }^{19}$. Dans ces conditions les rafles ne sont plus vertes et ne risquent plus de communiquer

16 X., Oec., 19.8. Pall. 22.16, évoque des pratiques permettant d'atténuer la brûlure du soleil, qui pourraient bien être grecques : envelopper les grappes dans des feuilles de figuier (que l'on rencontre souvent dans les vignes, dans des systèmes complantés) ou bien couvrir les grappes de feuilles de vigne. De même, la nuit, les feuilles de vigne pouvaient protéger les grappes de la rosée ou de la pluie.

17 Brun 2004, p. 91. Les raisins gâtés et les feuilles étaient scrupuleusement triés, et ceux qui foulaient le raisin devaient se laver les pieds et n'étaient pas autorisés à boire ni manger pendant le foulage (Geop. 6.11).

18 Amouretti 1996, 50-52.

19 Ap. Geop. 5.45.3. Dans cet extrait, (5.45) Diophanès évoque d'autres méthodes pour évaluer la maturité du raisin (on parle d'une $\delta \circ \kappa ı \alpha \sigma \sigma \alpha)$. L'une d'elles consiste à enlever une baie. Si l'espace laissé libre ne diminue pas les jours suivants, alors les baies sont mures et ne s'accroissent plus (Geop. 5.45.5). Sur la façon d'apprécier la maturation du raisin dans le monde romain, cf. Billiard 1913, p. 428, avec références. 
au moût un goût herbacé. Les Grecs notaient justement que les pépins noirs sont un bon indicateur de la maturation du raisin ${ }^{20}$.

Le foulage qui suivait libérait le jus des grappes et permettait aux pellicules de baigner dans le moût, ce qui assurait une colonisation homogène du milieu par les levures. Ce prolongement du contact entre pellicule et moût était aussi primordial pour le potentiel aromatique du futur vin. La pratique du foulage à la vigne, qui provoquait, avant l'entonnage dans les pithoi, un démarrage précoce du processus fermentaire, comportait des risques. Le foulage de raisins passerillés était une opération longue - plusieurs heures -, avec un moût solide et un jus très dense. Ces moûts très denses connaissaient des fermentations lentes et avaient une faible acidité, en particulier peu d'acide malique, et une quantité moindre de bactéries acétiques et lactiques. Il n'y avait donc pas de fermentation malolactique après la fermentation alcoolique. Le passerillage contribuait en revanche à l'augmentation de l'acide citrique, dont la présence est essentielle à l'équilibre des vins, et contribue à préserver une impression de fraicheur. Comme le notait Marie-Claire Amouretti, l'utilisation du pressoir et donc la production d'un vin de presse ne semble pas avoir été très fréquente dans le monde $\operatorname{grec}^{21}$. Dans tous les cas, le «vin de presse » n'est jamais un vin de qualité22.

Les Grecs ne pratiquaient pas la macération du moût avec le marc : les vins rouges tels que nous les connaissons aujourd'hui n'existaient donc pas ${ }^{23}$. Le Thasos et le Maronée sont néanmoins souvent qualifiés de vins rouges ou de vins noirs ${ }^{24}$. Comment expliquer ce paradoxe ? On peut tout de suite

20 Geop. 5.45 .2 (Diophanès d'après Démocrite et Africanus). Il est également spécifié que le raisin est à son point de maturé idéale pendant six jours seulement, ce que l'expérience confirme. Voir également Pl., HN, 10.11 et Col., Rust., 11.2.

21 Amouretti 1996, 52-53.

22 Comme le souligne Salviat 1986, p. 175, n. 33.

23 Voir les remarques, dans le même sens, de Brun et Tchernia 2004, 238-239. La distinction qu'opère Amouretti 1996, p. 50, doit être abandonnée.

24 Pour le Thasos, voir les testimonia reunis par Salviat 1986, p. 177-178. Théopompe ap. Ath. $1.26 \mathrm{~b}$, indique ainsi que les habitants de Chios ont été les premiers à produire un vin noir. 
écarter l'hypothèse de cépages teinturiers, c'est-à-dire de cépages à jus rouges, à faible teneur en sucres naturels, et donc incompatibles avec la production de vins moelleux. Même dans le cas de cépages à peau rouge, le passerillage préalable à l'élevage des vins moelleux de Thasos et de Maronée ne permettait pas une grande extraction de matière colorante, en particulier les anthocyanes présents sous la peau, peu solubles dans le moût et dans l'eau. La coloration ambrée de ces vins ne peut donc s'expliquer que par la macération pelliculaire dans l'aire de foulage et au moment du foulage ou bien par l'élevage des vins en milieu oxydatif, j’y reviendrai. Le Thasos et le Maronée devaient donc, après filtration, avoir une robe proche des rivesaltes ambrés ou des vins grecs moelleux issus de vendanges passerillées. Cette coloration est directement liée à l'action d'une enzyme, la tyrosinase, qui catalyse l'oxydation des matières colorantes et des tanins et entraîne un changement de couleur du jus vers les couleurs dorées profondes. Ranger le Thasos ou le Maronée dans la catégorie des vins rouges, c'est donc entretenir une véritable confusion sur la nature de ces crus renommés ${ }^{25}$. D'une manière générale les jeunes vins étaient peu colorés. Pline confesse d'ailleurs : « nous savons qu'on ajoute (au vin) pour le teinter des substances colorantes, comme une sorte de fard pour le vin et pour qu'il s'en trouve ainsi épaissi $\gg^{26}$. Palladius évoque notamment les baies de myrtilles, colorant puissant ${ }^{27}$.

\section{Transvasement dans les pithoi}

Après le foulage, le moût était transvasé dans les jarres, les pithoi, qui étaient enterrés à moitié ou aux deux tiers selon la qualité du vin : « les pithoi doivent être placés dans un endroit sec, car si le finage produit un vin chétif, léger et atrophié, les pithoi seront enterrés aux deux tiers, et si elle produit un

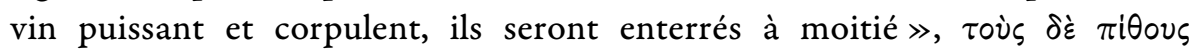

25 Cette question semble embarrasser Salviat 1986, lorsqu'il aborde la couleur et la qualité des vins thasiens.

26 Pl., $H N, 14.130$.

27 Pall. 11.14. 


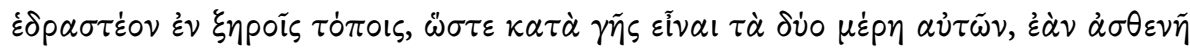

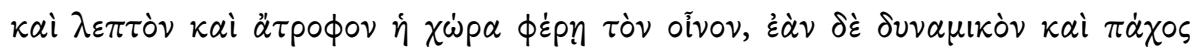

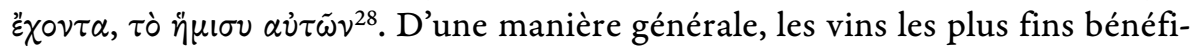
ciaient des contenants les plus petits ${ }^{29}$, l'enjeu étant la thermorégulation du moût tout au long du processus fermentaire ${ }^{30}$. Le cellier, ó oĩkos ó í $\pi \circ \delta \varepsilon \chi \dot{o} \mu \varepsilon v \circ \varsigma$

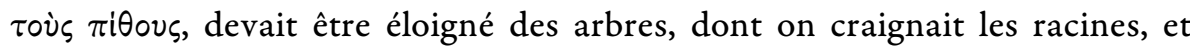
séparés des étables, des greniers et des bains ${ }^{31}$. Florentinus recommandait de bien séparer les jarres les unes de autres ${ }^{32}$. Si l'une d'elles venait à s'aigrir, il s'agissait d'éviter que les jarres voisines soient aussi affectées. Ces précautions devaient en effet permettre que de mauvaises souches de levures ne compromettent l'ensemble des vinifications, néanmoins, elles étaient très insuffisantes pour empêcher leur dissémination ${ }^{33}$.

Les jarres n'étaient pas complètement remplies, pour éviter que le moût en fermentation ne se répande dans le cellier. Pendant une période allant de 5 à 15 jours, les jarres restaient ouvertes et faisaient l'objet d'une surveillance attentive. Diophanès de Bithynie indique ainsi que l'ensemble des pellicules, pépins et autres matières solides du raisin qui montent à la surface du moût en fermentation devaient être prélevés quotidiennement pendant 15 jours, à la

28 Florentinus, ap. Geop. 6.2.3. Les pithoi de vins de faible qualité peuvent n'être enterrés qu’à moitié. Sur le vocabulaire employé pour décrire les qualités organoleptiques du vin, je me permets de renvoyer à ma contribution sur la dégustation du vin en Grèce ancienne, Boulay à paraître.

29 Anatolios, ap. Geop., 6.3.9, cf. Amouretti 1996, p. 53. Selon Anatolios, le moût qui fermente dans de larges pithoi perdent les arômes et leur bouquet est plus sujet à la mauvaise fleur (sur ce point, cf. infra).

30 Voir les expérimentations menées au Mas des Tourelles à Beaucaire, A. Tchernia dans Tchernia \& Brun 1999, p. 110.

31 Geop. 6.2.7 : « le cellier devrait être écarté des étangs, des écuries, des latrines, des tas de

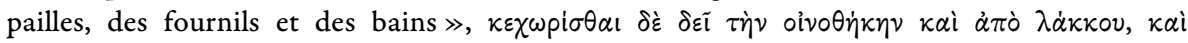

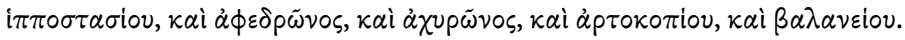

32 Geop. 6.2.2.

33 Les consignes d'hygiène concernant le chai témoignent des précautions qui entouraient les jarres en fermentation (Geop. 6.1, 2 et 10). 
main ${ }^{34}$. Dans certains cas, différents produits étaient ajoutés au moût avant sa fermentation complète et la fermeture des jarres. Il importe en effet d'insister tout particulièrement sur un point capital: les Grecs, qui ignoraient quel usage l'on pouvait faire du dioxyde de soufre, n'étaient pas en mesure d'empêcher le développement de micro-organismes aérobies, c'est-à-dire les bactéries, les moisissures et les levures qui se développent en présence d'oxygène. À la différence des Romains, les Grecs ne pratiquaient pas l'ouillage, usage consistant à remettre les récipients à niveau, encore inconnu des auteurs des Géoponiques. Les vins connaissaient donc un élevage oxydatif, avec de l'air présent au-dessus du vin. On parle parfois de vins sous atmosphère oxydative.

Cherchant à maîtriser empiriquement ce processus fermentaire périlleux, les Grecs ont utilisé différentes méthodes, selon les exigences du transport ou quand les circonstances l'imposaient :

1) L'addition de gypse chauffé ( $\gamma \dot{v} \psi \omega \sigma \iota \varsigma)$ - ou sulfate de chaux, on parle souvent de plâtrage - provoquait une importante augmentation de l'acidité réelle ${ }^{35}$. L'ajout de gypse diminuait le $\mathrm{pH}$ et contribuait ainsi à limiter le développement de bactéries ${ }^{36}$. Fronton préconisait le plâtrage du moût : celuici assure au vin sa durée, l'empêche de s'aigrir, tandis que la saveur piquante qu'il lui donne, disparaît au bout d'un certain temps ${ }^{37}$. Selon Palladius, cet usage, qui permettait de clarifier les vins ${ }^{38}$, n'était pas systématique ${ }^{39}$. En son

34 Geop. 6.12.

35 Les $\Gamma \varepsilon \omega \rho \gamma \iota \kappa \dot{\alpha}$ de Didymos (IV ${ }^{\text {e }}$ s. ap. J.-C. ?) proposent un protocole pour l'ajout de gypse,

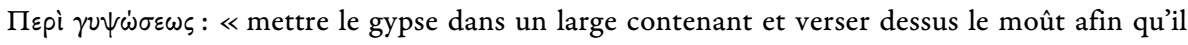
couvre le gypse; tournez-le bien et laissez ainsi jusqu'à ce qu'il soit reposé, afin que les morceaux de gypse les plus gros se précipitent au fond. Ensuite, retirez le moût du contenant, en prenant garde de ne rien emporter avec lui » (Geop. 6.18). Sur le plâtrage, voir également Billiard 1913, 499-500.

36 Kourakou-Dragona 1999, p. 138, n. 31. Voir également Brun et Tchernia 2004, p. 243.

37 Geop. 7.12.5.

38 Pall. 11.14.

39 Cf. Col., Rust., 12.19 : le vin supérieur n'a besoin de rien, et il ne faut pas altérer son goût naturel. Pl., $H N, 23.46$, était du même avis, pour des questions de salubrité. 
temps, Théophraste considérait lui cette pratique comme étrangère aux Grecs, et notait que cette coutume était en usage à Chypre, en Phénicie et en Italie ${ }^{40}$.

2) L'addition d'eau de mer ${ }^{41}$

On explique fréquemment l'ajout d'eau de mer par la nécessité de stabiliser les vins, en particulier les vins destinés à l'exportation ${ }^{42}$. Cette pratique avait d'autres visées et Plutarque consacre une question de ses Étiologies naturelles à ce sujet (Q. 10) : «Quant à l'eau de la mer, le sel qu'elle contient subtilise et dissout les substances hétérogènes qui se trouvent dans le vin, et empêche qu'il ne contracte une odeur désagréable ou un goût fade. D'ailleurs, les parties terreuses et grossières du vin s'attachant à ces corps étrangers et pesants qu'on y mêle, la lie se précipite au fond (de la jarre), et le

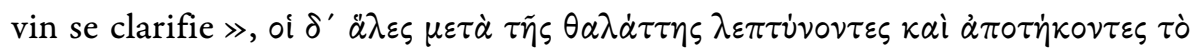

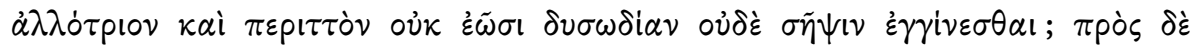

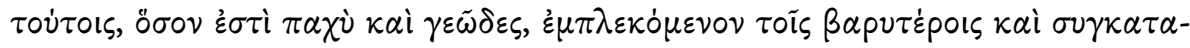

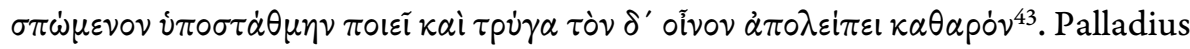
indique également que salage permettait d'obtenir des vins plus limpides, plus brillants, moins sensibles à la maladie de la tourne et à l'acétification ${ }^{44}$.

Les modernes qui ont commenté les textes préconisant cette addition de sel évoquent avant tout un moyen de conservation. C'est l'explication qui vient le plus naturellement, puisqu'effectivement le salage remplit ce rôle, en stoppant la croissance des micro-organismes, prévenant ainsi la formation d'une mauvaise fleur (cf. infra) ${ }^{45}$. Toutefois, les sources laissent dubitatif car

\footnotetext{
40 Thphr., Lap., 9.67.

41 Voir à ce sujet les remarques de Billiard 1913, 500-502. Pl., HN, 10.78, affirmait que l'addition d'eau de mer était née d'une fraude d'un esclave de Cos qui dissimulait ainsi ses prélèvements liquides.

42 E.g. Amouretti 1996, 55-56.

43 Plu., Mor., 914d-e. Traduction de l'Abbé D. Ricard légèrement modifiée.

44 Pall. 11.14. Voir A. Tchernia dans Tchernia \& Brun 1999, p. 114, sur l'effet clarificateur du sel, constaté par François Turié, préparateur à l'École de pharmacie de Montpellier et auteur d'une thèse sur la pratique du salage soutenue et publiée en 1894.

45 Col., Rust., 12.23.
} 
elles indiquent que les additions se limitaient à 2 gr par litre, au maximum ${ }^{46}$. Démocrite recommande seulement $0,5 \mathrm{gr}$ par litre, et dans le seul cas d'une vendange affectée par la pluie ${ }^{47}$. À la fin du XIX ${ }^{e}$ s., alors que la pratique du salage était assimilée à une fraude, on estimait qu'il fallait au moins 3 grammes de sel par litre de vin pour qu'une dégustation révélât le salage ${ }^{48}$. La loi du 11 juillet 1891 tolérait le salage jusqu'à 1 gr par litre. Cette concentration parait assez faible pour jouer ce rôle. Mais le vin est déjà un milieu relativement stable, non seulement à cause de l'alcool mais aussi d'autres produits du métabolisme des levures et bactéries. En outre, les éléments les plus fermentescibles ont été fermentés. Alors il est possible que de telles concentrations de sel suffisent (en synergie avec le reste) à améliorer la conservation ${ }^{49}$. En tout cas il ne semble pas qu'il ait été utilisé pour changer le goût du vin: Columelle recommande de faire des essais pour savoir quelle quantité rajouter pour ne pas « offenser le goût $\gg^{50}$.

3) Méthodes pour diminuer l'acidité

Lorsqu'il s'agissait de diminuer l'acidité d'un vin, ce qui arrivait rarement, les Anciens avaient la possibilité d'ajouter du marbre pulvérisé ou du calcaire argileux ${ }^{51}$.

46 Voir A. Tchernia dans Tchernia \& Brun 1999, p. 114, sur le salage des moûts pratiqué dans le monde romain occidental, tout aussi limité que dans le monde grec.

47 Geop. 7.4. Sur l'identité de ce Démocrite, voir Georgoudi 1990, 59-60.

48 Biquet 1900, p. 52.

49 Ces remarques sur le salage m'ont été communiquées par A. Lonvaud au cours de nos échanges sur les techniques de vinifications, je l'en remercie vivement. Mme Lonvaud me fait d'ailleurs remarquer à propos de l'utilisation de chlorure de sodium: «nos contemporains vinificateurs savent qu'ils peuvent en attendre une amélioration gustative, mais la règlementation l'interdit », constat qui tranche avec l'interprétation généralement très négative de cette pratique par les modernes. Ainsi pour A. Bresson 2007, p. 133, le salage des vins se fait « nécessairement » au détriment de leur qualité.

50 Col., Rust., 12.21.5. Comme le soulignait Amouretti 1996, p. 55, « le sel était naturellement intégré dans un certain nombre de vins des îles par l'apport de la brume marine ».

51 Voir Billiard 1913, 502-503, avec renvois à Caton, Columelle et Pline. 


\section{Fermeture des jarres/couverture du vin}

Après les premiers jours de fermentation, les jarres étaient fermées. Les Géoponiques précisent que les Grecs avaient coutume de placer sur le moût en fermentation, avant fermeture des pithoi, « une couronne de feuilles de menthe, d'origan ou de calament $\gg^{52}$. Dans son étude du livre IV de Pietro de' Crescenzi consacré aux techniques vinicoles, largement inspiré des Géoponiques, Jean-Louis Gaulin remarquait que ces plantes appartiennent toutes à la famille des lamiacées, mais restait dubitatif quant à l'action réelle de celles$\mathrm{ci}^{53}$. La recherche récente permet d'expliquer la pertinence de cette pratique. Ces plantes contiennent des phénols qui ont des propriétés aromatiques mais également antibactériennes et antifongiques, ainsi que des terpènes (une classe d'hydrocarbures) volatils, présents dans les huiles essentielles, aux propriétés antiseptiques ${ }^{54}$. Les Grecs avaient empiriquement compris l'emploi qui pouvait être fait de ces plantes. Leurs composés antimicrobiens agissaient sur les levures et ralentissaient le processus de fermentation.

Le vin placé dans des jarres enterrées ne connaissait pas de soutirage. Les vins étaient donc élevés sur lies. Le rôle antioxydant des lies est aujour-

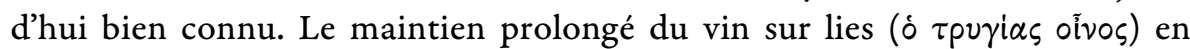
jarre autorisait des élevages oxydatifs tout en préservant les arômes fruités du vin.

$\mathrm{Au}$ moment de la fermeture des jarres, la fermentation alcoolique était déjà bien avancée. Elle pouvait se prolonger encore plusieurs semaines, selon la quantité de sucres résiduels présents. À la fin de l'hiver, quand la température du cellier dépassait $10^{\circ} \mathrm{C}$, un voile de levure se développait à la surface libre $\mathrm{du}$ vin au contact de l'air. On appelait ce voile la fleur du vin. Au contact de l'air les micro-organismes aérobies se développent et s'unissent, forment un voile très fin qui petit à petit s'épaissit. Ce voile connaît une régénération

52 Florentinus ap. Geop. 6.14.

53 Gaulin 1984, en particulier p. 118.

54 Je remercie A. Lonvaud pour ses éclaircissements sur ce point. 
constante. Les levures mortes sédimentent au fond de la jarre et subissent le processus d'autolyse.

Si le voile était constitué de bonnes levures, en particulier de Saccharomyces cerevisiae, la fleur n'était pas dangereuse pour le vin, il conférait au contraire à celui-ci des caractéristiques analytiques et organoleptiques spécifiques. On peut citer, au nombre des mécanismes qui entraient alors en jeu, le développement d'arômes caractéristiques des vins de voile, en particulier l'arôme de pomme, que le comique Hermippos présente comme caractéristique du vin de Thasos ${ }^{55}$. Il pouvait cependant se former un voile d'une couleur et d'une texture différente annonçant une détérioration sérieuse du vin causée par des bactéries acétiques.

Les Grecs connaissaient ce risque et recommandaient une surveillance accrue au moment où était susceptible de se former la fleur du vin, en particulier après l'équinoxe de printemps ${ }^{56}$. Ainsi, Sotion, peut-être le philosopheparadoxographe, actif au début du $\mathrm{I}^{\mathrm{er}}$ s. ap. J.-C. ${ }^{57}$, dans un chapitre concernant les pronostics sur la question de savoir si le vin tournera ou non, indique que l'on a l'habitude de juger le vin « également à cette fleur qui surnage en surface. $S$ 'il se produit une fleur large et souple tirant sur le pourpre, le vin est plus sûr ; si la fleur est gluante, ce n'est pas bon. La fleur noire ou blonde surnageant à la surface du vin montre un vin faible, la blanche, un vin stable ;

55 Ap. Ath. 1.29 e-f, cf. Kourakou-Dragona 1999, 122-124.

56 Kourakou-Dragona 1999, 110-111. Un fragment du poète Alexis (Ath. 2.36e) qui compare les étapes de la vinification à la mutation du tempérament humain, de la jeunesse à la vieillesse (voir à ce sujet l'étude de Mauduit 2002, à laquelle j'emprunte la traduction qui suit), évoque la fleur comme un véritable rite de passage entre la jeunesse et l'âge adulte du vin, la folie qui couvre sa surface disparaît et faire place au calme : «En effet, le vin jeune doit, de toute nécessité, comme l'homme, cesser d'abord d'être bouillant et insolent, puis, sa fleur perdue, devenir sec, puis, quand ses forces déclinent et que, purifié de tout ce dont je parle, il perd cette folie qui couvrait sa surface, devenir enfin potable, retrouver son calme et rester désormais

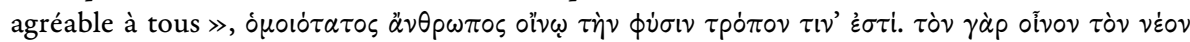

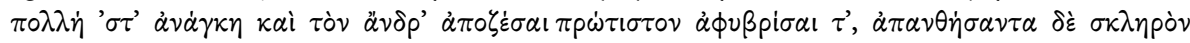

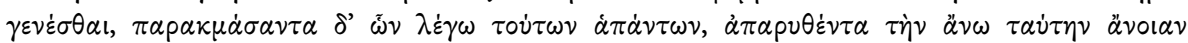

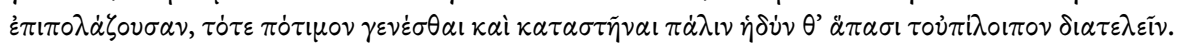
57 Georgoudi 1990, 56-57. 
quant à la fleur de type araignée, c'est le présage d'une altération immédiate »,

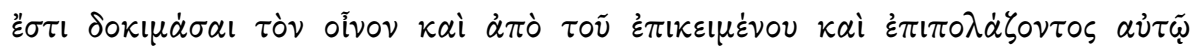

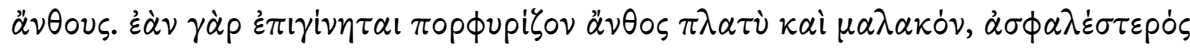

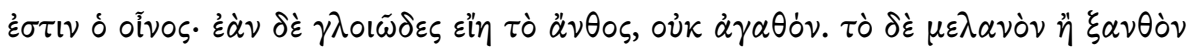

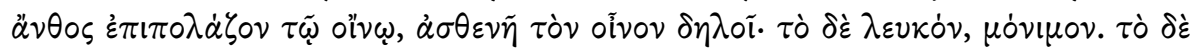

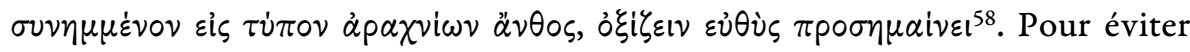
cette situation, Columelle suggérait de tenir le vin au frais ${ }^{59}$. En cas de problème, il était nécessaire de transvaser le vin, opération longue et délicate - il fallait en effet tirer le vin par le haut -, ce qui présentait l'avantage de le rafraichir et de l'aérer, et le faire fermenter de nouveau sur des lies de bon $v^{v i n}{ }^{60}$. Par ailleurs, le poissage préalable des pithoi avant l'entonnage, qui semble être devenu peu à peu systématique ${ }^{61}$, permettait de détruire les bactéries acétiques présentes dans le milieu ${ }^{62}$. Un passage de la Synagogè d'Anatolios de Bérytos (compilation du IV ${ }^{\mathrm{e}}$ s. ap. J.-C.), repris dans les Géoponiques, indique par ailleurs que l'utilisation de pithoi de plus petite capacité permettait de diminuer les risques de développement d'une mauvaise fleur ${ }^{63}$.

\section{Ouverture des jarres et séparation du vin de la fleur}

Traditionnellement, on ouvrait les pithoi au mois Anthestérion, le mois de mars, en particulier au moment de la plus ancienne des fêtes en l'honneur de Dionysos, les Anthestéria, du 11 au 13 Anthestérion. Il est souvent admis que le nom de cette fête populaire, la « fête des fleurs », est en lien avec la

\footnotetext{
58 Geop. 7.15.6.

59 Col., Rust., 12.30. Cf. Pall. 9.17.

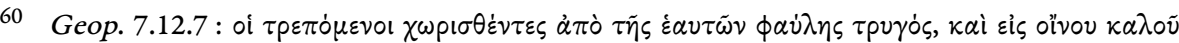

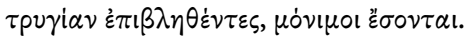

61 Amouretti 1996, p. 53, note justement que la moitié du livre VI des Géoponiques est consacrée à la préparation de la poix, à la fabrication des pithoi et à leur vernissage.

62 Brun 1991, p. 86.

63 Geop. 6.3.9. Sur ce recueil et son intégration dans les Géoponiques, voir la contribution essentielle de Guignard 2009.
} 
renaissance du monde végétal ${ }^{64}$. Les noms de la fête et du mois sont en effet construits sur le mot fleur, $\tau \dot{\partial} \ddot{\alpha} \nu \theta \circ \varsigma$ et le verbe fleurir, $\dot{\alpha} \nu \theta \varepsilon \tilde{\imath} v$. Cependant, le rapprochement n'est pas vraiment satisfaisant, puisque la fête avait lieu à la fin du mois de février ou au tout début du mois de mars. Je proposerais une autre interprétation : il me semble que le nom de cette fête se rapporte plus directement à la fleur du vin qui se développe à la sortie de l'hiver, et plus exactement à la délicate séparation du vin et de la fleur, au moment du transvasement du vin des jarres dans les amphores, «après le passage du zéphyr, car aucun vent n'altère et ne fait tourner le vin autant que celui-ci et l'on considère que le vin qui a résisté à son influence se conserve ensuite intact $\gg^{65}$. La cérémonie débutait par l'ouverture des jarres, la $\pi i \theta \circ / \gamma i \alpha^{66}$, suivie d'un symposion public. En Attique, le premier jour des Anthestéries, chaque vigneron apportait une jarre de vin nouveau près du sanctuaire de Dionysos dans les marais - sans doute en bordure de l'Ilissos -, pour offrir une libation au dieu de la vigne ${ }^{67}$.

Avant de goûter le vin nouveau, il fallait d'abord puiser le vin. Une journée de repos était nécessaire pour ensuite boire le vin transvasé, le deuxième jour, en particulier si l'on procédait à un collage au blanc d'œufs ${ }^{68}$. D'autres additions permettaient de stabiliser les vins tirés des pithoi. Le fenu-

64 Il n'est pas dans mon propos de reprendre l'ensemble des interprétations qui ont été avancées. Voir en dernier lieu Noël 1999.

65 Plu., Mor., 655e. Noël 1999, p. 129, note justement à propos de ce passage, que « la date de la fête se rapporte au processus même de la vinification ».

66 Noël 1999, p. 130 : « Ouvrir les jarres marquait la fermeture de la parenthèse commencée avec la vendange et achevée avec la précipitation des dernières lies $\gg$.

67 Ath. 11.465a (Phanodème, FGrHist, 325 F 12 Jacoby) : « Au sanctuaire de Dionysos du Marais, les Athéniens apportaient le vin doux des jarres et en mélangeaient pour le dieu et ensuite en prenaient pour eux-mêmes. C'est pour cela que Dionysos était appelé dieu du Marais, parce que le vin doux était mélangé et bu avec de l'eau cette occasion pour la première fois. C'est pourquoi les Nymphes étaient appelées aussi les nourrices de Dionysos parce que l'eau augmente le vin quand il est mélangé avec elle.» (trad. Noël 1999, p. 131, avec commentaire).

68 Geop. 7.22; Pall. 11.14. Voir Billiard 1913, 508-509. 
grec, qui contient de grandes quantités d'acide ascorbique, aux propriétés antioxydantes, était ainsi employé pour empêcher le vin de tourner (graines séchées puis écrasées) ${ }^{69}$.

Le célèbre stamnos attique à figure rouge des années 420 conservé au Musée archéologique de Naples (fig. 1$)^{70}$, attribué au peintre du Dinos de Berlin, illustre, comme l'a montré Stavroula Kourakou-Dragona, de façon très réaliste cette cérémonie de transvasement du vin «fleuri »: «des femmes figurées en ménades ont dressé une table devant l'effigie de Dionysos, habillée et ornée de feuilles de lierre, de branches et de fruits. Sur la table, deux stamnoi semblables, un canthare - verre sacré de Dionysos - et des morceaux épars de «fleur » blanche, que le peintre a rendus par des touches de blanc. Il a même évoqué la liquidité de la « fleur » qui s’est écoulée, salissant la table. Ces ménades - les femmes initiées - exécutent une danse sacrée, à l'exception de l'une d'elle qui transvase le vin : puisant avec une arytaina qu'elle tient à la verticale, un instrument propre à puiser le vin, dans un premier stamnos, elle remplit un skyphos avec lequel elle transporte le vin, désormais propre, dans le stamnos voisin. Toute son attention est absorbée par la tâche délicate qu'elle est en train d'exécuter. $\gg^{71}$

Quand la fleur recouvrait le vin, il fallait puiser ( $\dot{\alpha} \dot{\omega} \omega)$ le vin limpide sous la fleur, et pour ce faire, on utilisait un ustensile particulier, l'ápí $\tau \alpha \iota \nu \alpha^{72}$. Cet usage s'est conservé jusqu'à nos jours dans une région qui pratique encore l'élevage sous voile, à Jérez où l'on emploie la venencia, manipulée avec habileté

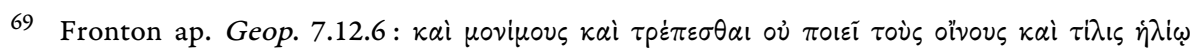

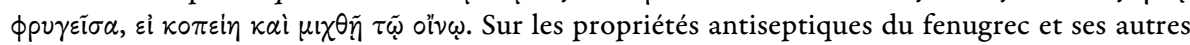
usages (notamment pour parfumer les vins ou leur donner le caractère de vins vieux), voir également A. Tchernia dans Tchernia \& Brun 1999, p. 115 et 139-140.

70 Mus. Naz. 81674 (H Naples 2419; Beazley 1963, p. 1151, n 2), dit de Nocera (peut-être de Nora). Sur ce vase, voir en particulier Elia 1963-1964.

71 Kourakou-Dragona 1999, p. 112.

72 Ath. 10.424b. Elia 1963-1964 notait d'ailleurs que l'on avait retrouvé une arytaina dans la même tombe, identique à celle représentée sur le stamnos, et supposait que le défunt pouvait être un myste de Dionysos. La Genière 1987, 57-58, pense à « une femme initiée qui aurait eu une fonction dans le rituel ». 
par le venenciador. Le venenciador tient l'ustensile par le haut du manche, de la même manière que les échansons tenaient l'arytaina. Lorsque la venencia est plongée dans le liquide, on la retire brusquement pour la remplir de vin propre.

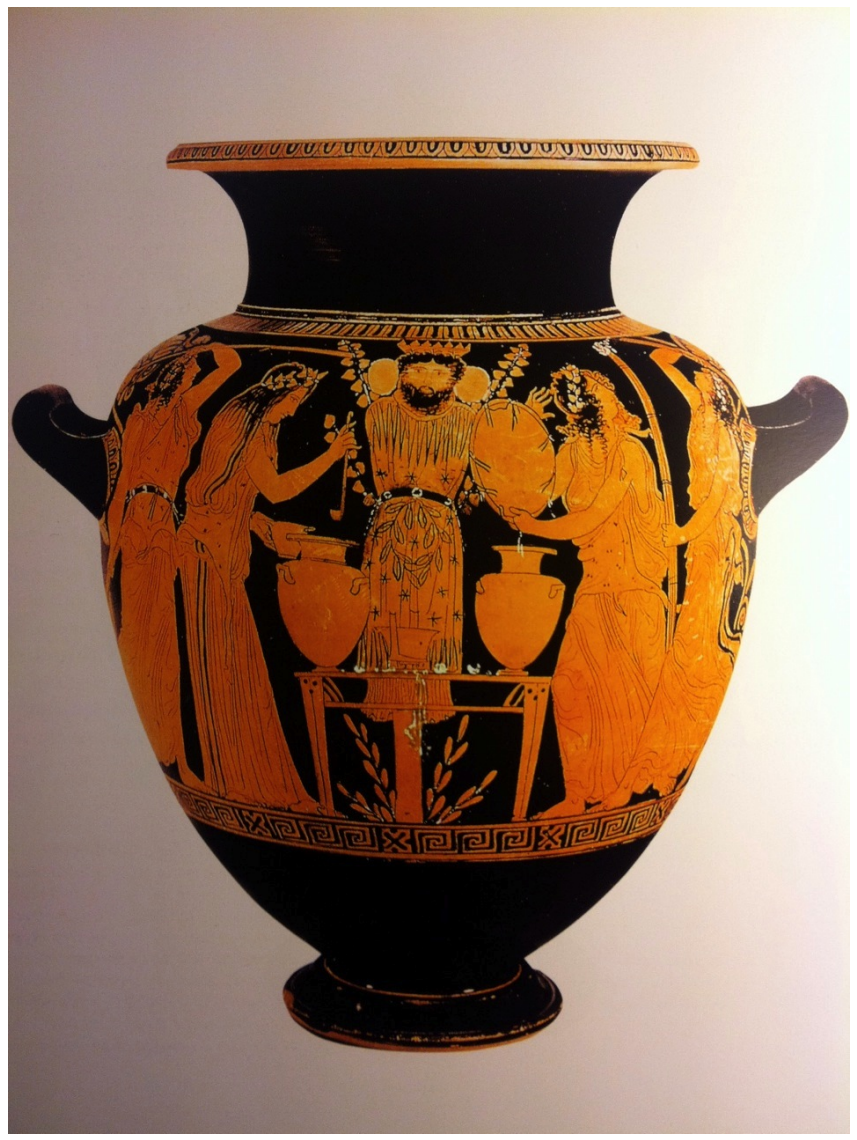

Fig. 1 : Le stamnos de Nocera (Museo Archeologico Nazionale). Source : Kourakou-Dragona 1999, p. 113 
Si l'on reconnaît communément dans le décor de ce stamnos l'illustration d'une cérémonie dionysiaque, les chercheurs se divisent, depuis le début du siècle, sur la question de savoir s'il faut relier ce vase et le groupe des vases à figures rouges décorés de scènes dionysiaques (70 environ) à la fête des Anthestéries ou à la fête des Lénéennes, comme le proposait August Frickenhaus $^{73}$. Et si l'on doit rapprocher le stamnos de Nocera d'une fête en l'honneur de Dionysos, ce ne peut être que les Anthestéries, en raison de la présence de la fleur. Lors des Lénéennes, en décembre-janvier, la présence de la fleur à la surface du vin est inenvisageable, en raison de la température dans les celliers.

Il convient d'adopter une grande prudence concernant ces Lenäenvasen, et il est sans doute préférable, comme l'a montré Françoise FrontisiDucroux $^{74}$, de voir dans ces représentations une évocation des fêtes dionysiaques en général. Cependant, il me semble que dans le cas présent, le souci du détail du peintre permet de rapprocher cette scène des délicates opérations qui accompagnaient l'ouverture des pithoi au premier jour des Anthestéries ${ }^{75}$.

\footnotetext{
73 Frickenhaus 1912, p. 1-40, pl. 1-5. Voir La Genière 1987.

74 Frontisi-Ducroux 1991 et 1997.

75 La mise en lumière de cette pratique permet, je crois, de reprendre un point du commentaire d'une inscription publiée par Salviat 1958 (SEG 17, 415), donnant la liste des jours où on ne pourra pas faire telle ou telle procédure. Dans la liste, figure les Anthestéries avec

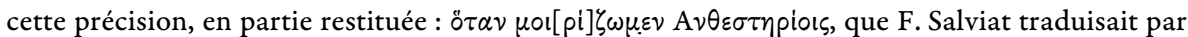
« lorsque nous procédons au partage, aux Anthestéries ». J. \& L. Robert, Bull. 1959, 323, p. 227 notaient : «ces mots restent obscurs 》. Selon Salviat 1958, 227-228, ces mots renvoyaient à un partage du vin, peut-être à une «répartition » de la récolte, « sous contrôle officiel », au moment des Anthestéries. Je croirais volontiers que cette précision renvoie en réalité à la séparation du vin et de la fleur, au premier jour des Anthestéries, et je proposerais de traduire « lorsque nous procédons à la séparation ». La forme inédite, proposée par F. Salviat en raison de la lacune, qui selon lui, « doit être remplie par une lettre ordinaire + iota » (Salviat 1958,

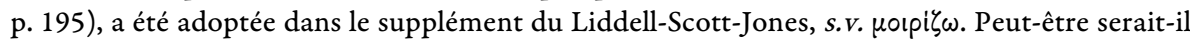

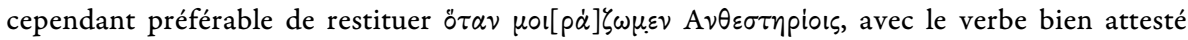
$\mu \circ 1 \rho \dot{\alpha} \omega$.
} 
Les 2 et 3 juin 2008, près de 150 ans après la publication des Études sur le vin de Louis Pasteur, l'INRA Montpellier a accueilli une conférence internationale réunissant des scientifiques, des œnologues et des professionnels du secteur du vin de divers horizons, sur le thème de l'influence de l'oxygène sur le vin et sa prise en charge au cours des différentes étapes de la fabrication du vin. Tous les chercheurs se sont accordés à reconnaitre la complexité du sujet et se sont félicités des progrès réalisés dans ce domaine. Les vignerons grecs de l'Antiquité, qui ne disposaient d'aucune technique d'analyse ou de mesure et ignoraient l'usage du dioxyde de soufre, auraient certainement eu des choses à apprendre aux participants de cette conférence qui cherchaient à mieux comprendre l'influence de l'oxygène sur le développement des arômes, la formation du goût et de la structure ainsi que sur la couleur du vin. L'appréhension concrète de la nature des vins grecs laisse en effet entrevoir comment les techniques vinicoles élaborées de manière pragmatique par les Grecs encadraient les risques d'une vinification sous atmosphère oxydative et visaient plus particulièrement à contrôler l'oxydation du vin. On peut attendre des progrès d'une collaboration accrue avec les chercheurs en microbiologie, qui montrent toute la rationalité et le degré d'élaboration des pratiques grecques, qui ont souvent été qualifiées de recettes superstitieuses. D’autres avancées, très récentes, laissent espérer des progrès décisifs, en particulier pour la connaissance des cépages de l'antiquité. En 2007, le génome de la vigne a été entièrement décrypté. Dès 2008, il était possible de préciser les relations de parenté entre les cépages existants. Jusqu'à présent cependant, aucun résultat positif, en terme d'identification variétale, n'a été obtenu avec l'extraction et l'analyse d'ADN ancien sur vigne (que ce soit sur des pépins ou des fragments de sarment), mais cela ne saurait tarder. Quoi qu'il en soit de ce dernier point, cette approche pluridisciplinaire, qui n'est ici qu'esquissée, offre de nouvelles perspectives, bien au-delà des seules questions techniques, puisqu'elle permet de proposer de nouveaux éclairages sur les usages grecs liés au vin et à sa consommation et de préciser l'interprétation de représentations figurées sur les vases grecs. 


\section{Bibliographie}

Amouretti, M.-C. (1996) : "L’originalité technique du vin grec et les traditions en Méditerranée orientale”, Münsteriche Beiträge zur antiken Handelsgeschichte 15, 42-66.

Beazley, J. D. (1963) : Attic Red-Figure Vase-Painters, $2^{\mathrm{e}}$ éd., Oxford.

Billiard, R. (1913) : La vigne dans l'Antiquité, Lyon.

Biquet, J. (1900) : Manuel juridique et pratique de l'achat et de la vente du vin, MontpellierParis.

Boissinot, P. (2003) : "Métrologie de l'arboriculture antique dans le Midi de la France", in : Favory 2003, 37-57.

Boulay, T. (à paraître) : “Tasting Wine in Ancient Greece”, in : Wilkins et Nadeau à paraître.

Bouvier, M. (2007), Les vins de l'Antiquité, Paris.

Braund, D. et J. M. Wilkins, éd. (2000), Athenaeus and his world. Reading Greek Culture in the Roman Empire, Exeter.

Bravo, B. (1997) : Pannychis e simposio, con uno studio iconographico di F. Frontisi-Ducroux, Pise-Rome.

Bresson, A. (2007) : L'économie de la Grèce des cités I. Les structures et la production, Paris.

Brun, J.-P. (1991) : "La viticulture en Provence", Gallia 58, 69-89.

Brun, J.-P. (2004) : Archéologie du vin et de l'huile, de la préhistoire à l'époque hellénistique, Paris.

Brun, J.-P., M. Poux et A. Tchernia, dir. (2004) : Le vin, nectar des dieux, génie des hommes, Lyon.

Brun, J.-P. et A. Tchernia (2004) : "Vendanges et vinifications", in : Brun, Poux et Tchernia 2004, 232-249.

Dalby, A., trans. (2010) : Geoponika: Farm Work. A Modern Translation of the Roman and Byzantine Farming Handbook, Totnes.

Dubourdieu, D., A. Lonvaud, P. Ribéreau-Gayon et B. Donèche (2004): Traité d'œnologie : Tome 1, Microbiologie du vin, vinifications, $5^{\mathrm{e}}$ éd., Paris.

Elia, O. (1963-1964) : "Lo stamnos dionisiaco di Nocera”, Apollo, III-IV, 79-92.

Empereur, J.-Y. et Y. Garlan (1986) : Recherches sur les amphores grecques, BCH Suppl. 13, 174175.

Favory, F., éd. (2003): Métrologie agraire antique et médiévale: actes de la table ronde d'Avignon, Besançon.

Frickenhaus, A. (1912) : Lenäenvasen, Programm zum Winckelmannsfeste der Archäologischen Gesellschaft zu Berlin, 72, Berlin.

Frontisi-Ducroux, F. (1991) : Le Dieu-masque, une figure du Dionysos d'Athènes, Paris-Rome. 
Frontisi-Ducroux, F. (1997) : “Retour aux vases des Lénéennes”, in : Bravo 1997, 127-132.

Gaulin, J.-L. (1984) : "Sur le vin au Moyen-Âge Pietro de Crescenzi lecteur et utilisateur des Géoponiques traduites par Burgondio de Pise”, MEFRA 96, 95-127.

Georgoudi, S. (1990): Des chevaux et des bœufs dans le monde grec : réalités et représentations animalières à partir des livres XVI et XVII des Géoponiques, Paris.

Gourevitch, D. (2000): "Hicesius' fish and chips. A plea for an edition of the Fragments and Testimonies of $\pi \varepsilon p i$ i $\nu \lambda \eta \varsigma^{\prime}$, in: Braund et Wilkins, 2000, chap. 37, 483-491.

Guignard, C. (2009): "Sources et constitution des Géoponiques à la lumière des versions orientales d'Anatolius de Béryte et de Cassianus Bassus", in : Wallraff et Mecella 2009, 243344.

Jouanna, J. (1996) : "Le vin et la médecine dans la Grèce ancienne”, REG 109, 410-434.

Jouanna, J. et L. Villard, éd. (2002) : Vin et santé en Grèce ancienne, BCH Suppl. 40.

Kourakou-Dragona, S. (1999) : Un cratère rempli d'euphorie, Athènes.

La Genière, J. de (1987) : "Vases des Lénéennes ?”, MEFRA 99, 43-61.

Lonvaud-Funel, A., V. Renouf et P. Strehaiano (2010) : Microbiologie du vin : bases fondamentales et applications, Paris.

Mauduit, C. (2002) : "Les raisins de la colère : vin, humeurs et tempérament dans la littérature grecque", in : Jouanna et Villard 2002, 11-22.

Noël, D. (1999) : “Les Anthestéries et le vin”, Kernos, 12, p. 125-152.

Robert, J. et L. Robert (1989) : Claros I. Décrets hellénistiques, Paris.

Salviat, F. (1958) : "Une nouvelle loi thasienne : institutions judiciaires et fêtes religieuses à la fin du IV siècle av. J.-C.", $B C H$ 82, 1958, 193-267.

Salviat, F. (1986) : "Le vin de Thasos, amphores, vin et sources écrites", in : Empereur et Garlan $1986,145-195$.

Salviat, F. (1989) : "Les cépages de la vigne en Grèce ancienne", in : Les écrivains et le sacré : la vigne et le vin dans la littérature, Paris, 1989, 459-461.

Tchernia, A. (1997) : "Le cercle de L. Licinius Crassus et la naissance de la hiérarchie des vins à Rome", CRAI 141, 1247-1259.

Tchernia, A. et J.-P. Brun (1999) : Le vin romain antique, Paris, 1999.

Wallraff, M. et L. Mecella, éd. (2009) : Die Kestoi des Julius Africanus und ihre Überlieferung, Berlin-New York.

Wilkins, J. et R. Nadeau, éd. (à paraître) : Companion to Food in the Ancient World, Blackwell, Oxford. 